\title{
CORRELATION PROPERTIES OF QUANTIZATION NOISE
}

\author{
Peter Kabal \\ Electrical \& Computer Engineering \\ McGill University, Montreal, Canada \\ Peter.Kabal@McGill.CA
}

\begin{abstract}
This paper examines the correlation properties of quantization noise. The quantization noise energy is subtractive if the quantizer output levels are optimized for the probability density of the input signal (pdf optimized). This paper gives a new result that shows that a quantizer (uniform or not) which has quantizer break points midway between output levels (a minimum distance quantizer) and is scaled to minimize the mean-square error, also has this property. Examples are shown that show the correlation properties which determine whether the quantization noise energy is subtractive or additive. This paper also considers a postfilter configuration that compensates for the quantization noise. The postfilter frequency domain gains take the correlation properties of the quantization noise into account. An experiment on reducing the effect of quantization noise in speech gives an indication that taking account of the correlation is useful.
\end{abstract}

Index Terms - Quantizers, quantizer noise correlation

\section{INTRODUCTION}

Consider a scalar quantizer with an input $x$ and an output $y$. The quantization error is

$$
q=x-y .
$$

The quantization error (also referred to as the quantization noise) is often modelled as being additive in energy, i.e., the energy of the output signal is the sum of the input energy and the quantization error energy. However, the quantization error is a function of input value and so can be correlated with the input signal. One of the goals of this work is to elaborate on the correlation of the error with the signal.

One application of this work is to speech enhancement, i.e., reducing the perceptual impact of quantization noise using a postfilter. While the additive uncorrelated assumptions on the quantization error do lead to algorithms which improve speech quality, there are potential performance gains with better modelling of the effects of quantization noise.

This research was funded by a grant from the Natural Sciences and Engineering Research Council of Canada.

\section{PROPERTIES OF QUANTIZATION NOISE}

Consider a (one-dimensional) quantizer. The quantizer is specified by a set of $N+1$ break points $x_{q}[i]$ which define $N$ quantizer intervals. These break points are in increasing order. The first and last break points are fixed values, $x_{q}[0]=-\infty$ and $x_{q}[N]=\infty$. The $N$ output levels are $y_{q}[i]$, for $i=0, \ldots, N-1$. The input signal will be characterized by a probability density function $p_{x}(x)$.

\subsection{PDF-Optimized Quantizer Output Levels}

Given a set of quantizer break points, the output levels which minimize the mean-square error are the centroids of the intervals with respect to the probability density of the input signal,

$$
y_{q, \mathrm{opt}}[i]=\int_{x_{q}[i]}^{x_{q}[i+1]} x p_{x}(x \mid i) d x
$$

where $p_{x}(x \mid i)$ is the probability density function of $x$ when $x$ lies in the $i$ th interval. We will refer to a quantizer with the output levels chosen to be the centroids of the quantizer regions as having pdf optimized output levels.

It can be shown that the quantization noise is uncorrelated with the quantizer output [1],

$$
\sum_{i=0}^{N-1} \int_{x_{q}[i]}^{x_{q}[i+1]} y_{q, \mathrm{opt}}[i] q_{i}(x) p_{x}(x \mid i) d x=0 .
$$

Expressed as an expectation, a quantizer with pdf-optimized output levels has an error which is uncorrelated with the output,

$$
E[Q Y]=0 .
$$

This result can be used to show that the quantization error for the pdf optimized quantizer levels subtracts in energy,

$$
E\left[Y^{2}\right]=E\left[X^{2}\right]-E\left[Q^{2}\right] .
$$

The result developed here depends only on the quantizer output levels being optimized to minimize the mean-square error for a given set of quantizer break points. 


\subsection{Scaled Minimum-Distance Quantizers}

A minimum distance quantizer has quantizer break points which lie midway between output levels. The minimum distance property is a necessary requirement for the minimization of any error measure for which the measure increases monotonically with the error magnitude.

Consider a prototype minimum distance quantizer,

$$
x_{q}[i+1]=\frac{y_{q}[i+1]+y_{q}[i]}{2}, \quad 1 \leq i \leq N .
$$

Now scale the quantizer levels by $\alpha$ to minimize the meansquare error for a given input pdf. The mean-square error for the scaled quantizer is

$$
\varepsilon=\sum_{i=0}^{N-1} \int_{\alpha x_{q}[i]}^{\alpha x_{q}[i+1]}\left(x-\alpha y_{q}[i]\right)^{2} p_{x}(x) d x .
$$

If we take the derivative of this expression with respect to $\alpha$, we get three terms for each term in the sum. Two of these terms involve derivatives of the limits of the integrand. For a minimum distance quantizer, the expression involving the upper limit of one interval cancels the expression involving the lower limit of the adjacent interval. Then the sum of the derivatives over $i$ is

$$
\frac{d \varepsilon}{d \alpha}=2 \sum_{i=0}^{N-1} y_{q}[i] \int_{\alpha x_{q}[i]}^{\alpha x_{q}[i+1]}\left(\alpha y_{q}[i]-x\right) p_{x}(x) d x .
$$

Setting this derivative to zero, gives a parametric equation for $\alpha$. Rewrite the terms in Eq. (7) symbolically as

$$
\varepsilon=E\left[Q^{2}\right]=E\left[X^{2}\right]-2 E[X Y]+E\left[Y^{2}\right]
$$

Substituting the value of $\alpha$ into the expression for meansquare error, we find that $E[X Y]=E\left[Y^{2}\right]$ and finally that

$$
E\left[Y^{2}\right]=E\left[X^{2}\right]-E\left[Q^{2}\right]
$$

This is the same result as in Eq. (5) which was developed for pdf optimized quantizer output levels. The result for scaled minimum-distance quantizers is a generalization of the result for uniform quantizers given in [2].

There are two ways for the quantization error subtracting in energy for a given signal input signal. First, the quantizer output levels can be optimized for the pdf. Second, the quantizer break points satisfy the minimum distance property and the quantizer is scaled to minimize the mean-square error.

The two necessary conditions for a minimum meansquare error quantizer (pdf optimized output levels and minimum distance break points) can be applied alternately to iterate to a quantizer which minimizes the mean-square error. Reference [3] describes the Lloyd-Max algorithm which uses this iteration to design mean-square optimal scalar quantizers with non-uniform step sizes.

\subsection{Quantization Error Correlation}

We want to generalize the error expression Eq. (5) for different levels of correlation between the quantization error and the input signal. Write the mean-square value of the output $y$ as

$$
\begin{aligned}
E\left[Y^{2}\right] & =E\left[(X-Q)^{2}\right] \\
& =E\left[X^{2}\right]+\gamma_{x q} E\left[Q^{2}\right],
\end{aligned}
$$

where $\gamma_{x q}$ is a correlation parameter given by

$$
\gamma_{x q}=1-2 \frac{E[X Q]}{E\left[Q^{2}\right]} .
$$

Note that the correlation parameter scales the quantization noise energy. If the quantizer error energy is subtractive, $\gamma_{x q}=-1$. When the quantizer error energy is additive (quantization noise uncorrelated with the input signal), $\gamma_{x q}=1$.

\section{QUANTIZATION NOISE: EXAMPLES}

\subsection{Uniform Quantizer}

The first example is a symmetric uniform quantizer with the step size chosen to minimize the mean-square error for a signal with a unit variance uniform distribution. The signal-tonoise ratio (SNR) for these quantizers is plotted in Fig. 1 as a function of the signal variance.

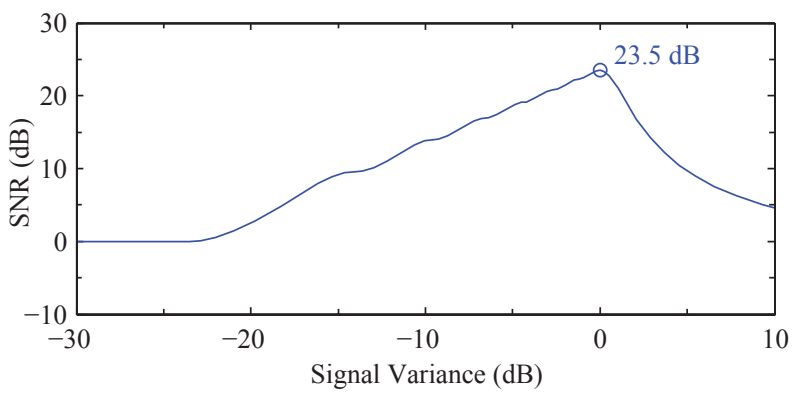

(a) SNR for a uniform quantizer with 15 levels

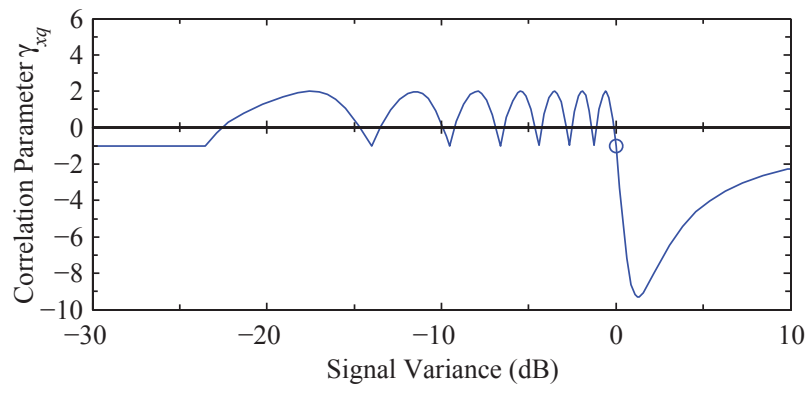

(b) Correlation parameter for a uniform quantizer with 15 levels

Fig. 1: Uniform quantizer acting on a uniform signal

The lower plot in Fig. 1 shows the correlation parameter $\gamma_{x q}$ defined in Eq. (12). The quantization noise is both positively and negatively correlated with the input signal. At those 


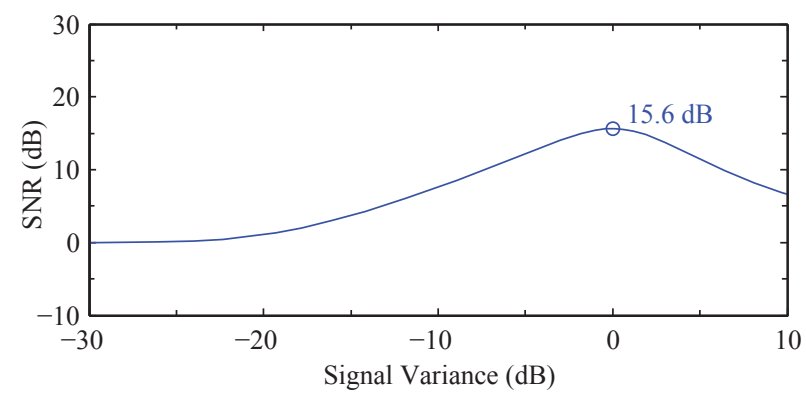

(a) SNR for a uniform quantizer with 15 levels

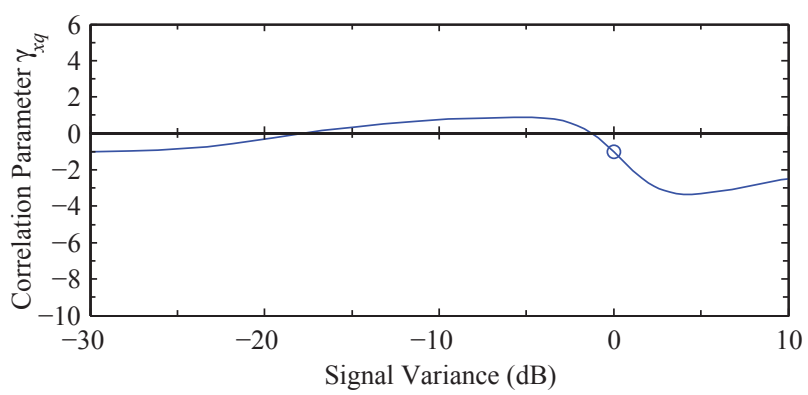

(b) Correlation parameter for a uniform quantizer with 15 levels

Fig. 2: Uniform quantizer acting on a Laplace signal

points where $\gamma_{x q}=-1$, the quantization error is completely power subtractive. Also at these points, the derivative of the SNR curve is zero. For other signal levels, the quantization noise can be additive or subtractive. For the uniform signal applied to a uniform quantizer, the pdf optimized condition and the minimum distance property are satisfied for several values of signal variance.

We now consider a signal with a two-sided exponential (Laplace) pdf. The SNR and correlation parameter plots are shown in Fig. 2. The uniform quantizer has 15 levels and has been designed to minimize the mean-squared error for a unit variance Laplace signal. We see the SNR plot is much smoother than seen earlier. The quantization error is subtractive for signal levels near $0 \mathrm{~dB}$, but is partially additive over a broad range of lower levels.

\subsection{Non-Uniform Quantizer}

The next comparison is for a mismatched non-uniform quantizer with the plots shown in Fig. 3. The quantizer is designed for a Gaussian signal, but has a Laplace signal applied. The quantizer itself has break-points which satisfy the minimum distance property. The correlation parameter is -1 at the point of maximum SNR. The quantization error is subtractive for signal levels near $0 \mathrm{~dB}$, but is partially additive over a broad range of lower levels.

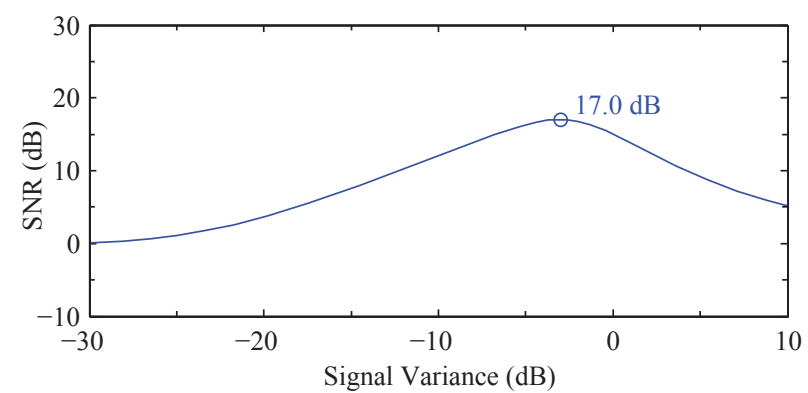

(a) SNR for a non-uniform quantizer with 15 levels

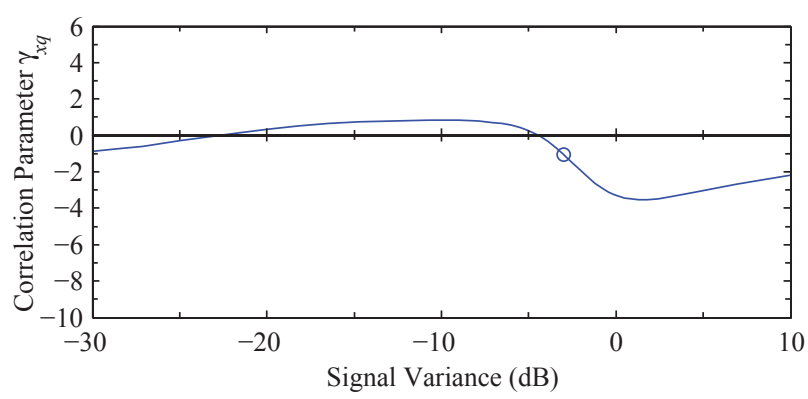

(b) Correlation parameter for a non-uniform quantizer with 15 levels

Fig. 3: Non-uniform quantizer (designed for a unit variance Gaussian signal) acting on a Laplace signal

\section{QUANTIZATION NOISE POSTFILTERING}

Speech enhancement is routinely used to reduce the perceptual impact of background noise prior to coding speech signals. In traditional speech enhancement, background noise levels are estimated during non-speech intervals. This estimation is carried out in the frequency domain. The amplitude of the estimated noise at each frequency is then subtracted from the amplitude of each frequency of the speech plus noise signal - this is simple spectral subtraction. A more general view of spectral subtraction is that of frequency-by-frequency gain modification.

Postfiltering is a form of speech enhancement applied in speech coding to help reduce the perceptual effect of coding noise. This is often accomplished using a pole/zero filter derived from the spectral envelope of the speech signal. In this application, the coding noise is strongly signal dependent. In the sequel we look at a simplified form of postfiltering, postfiltering to reduce the effects of quantization noise.

\subsection{Frequency Domain Postfilter}

Consider a postfilter implemented frame-by-frame in the frequency domain. Let the samples in the frame of length $N_{f}$ be $y[n]$. We explore the effect of noise correlated with the signal in the frequency domain. The DFT of quantized signal $y[n]$ 
after windowing is

$$
\begin{aligned}
Y_{w}[k] & =\sum_{n=0}^{N_{f}-1} w[n] y[n] W_{N_{f}}^{n k} \\
& =X_{w}[k]-Q_{w}[k] .
\end{aligned}
$$

where $W_{N_{f}}=\exp \left(-2 \pi / N_{f}\right)$. Then

$$
\begin{gathered}
E\left[\left|Y_{w}[k]\right|^{2}\right]=E\left[\left|X_{w}[k]\right|^{2}\right]-2 \operatorname{Re}\left(E\left[X_{w}[k] Q_{w}^{*}[k]\right]\right) \\
+E\left[\left|Q_{w}[k]\right|^{2}\right] .
\end{gathered}
$$

If the quantization noise $q[n]$ is a sequence with zero mean, independent, identically distributed values, we can simplify the last two terms and write the result in the same form as the time domain energy relationship ${ }^{1}$

$$
E\left[\left|Y_{w}[k]\right|^{2}\right]=E\left[\left|X_{w}[k]\right|^{2}\right]+\gamma_{x q} E\left[\left|Q_{w}[k]\right|^{2}\right],
$$

where the quantization noise energy per frequency bin is

$$
E\left[\left|Q_{w}[k]\right|^{2}\right]=E\left[q^{2}[n]\right] \sum_{n=0}^{N_{f}-1} w^{2}[n] .
$$

\subsection{Wiener Gain for Correlated Noise}

Let us find the real gain $g$ which minimizes the mean-square error,

$$
g_{\mathrm{opt}}[k]=\min _{g} E\left[|X[k]-g Y[k]|^{2}\right] .
$$

Taking the derivative with respect to $g$ and setting the result to zero, we can express the gain in terms of the correlation parameter

$$
g_{\mathrm{opt}}[k]=\frac{E\left[|X[k]|^{2}\right]+E\left[|Q[k]|^{2}\right]\left(\gamma_{x q}-1\right) / 2}{E\left[|X[k]|^{2}\right]+\gamma_{x q} E\left[|Q[k]|^{2}\right]} .
$$

We note that if $\gamma_{x q}=1$, we get the familiar Wiener gain for uncorrelated noise. If $\gamma_{x q}=-1$, the gain becomes unity. This occurs whenever the quantization noise is energy subtractive, i.e., the quantizer output levels are pdf optimized and/or the quantizer is minimum distance and scaled to minimize the mean-square error.

\subsection{Experimental Postfilter}

We implemented a simple postfilter acting on a speech signal ( $8 \mathrm{kHz}$ sampling rate) which was quantized with a 63 level uniform quantizer (designed for a \pm 1 input range. The input signal was divided into frames of 128 samples $(50 \%$ overlap). A square root sine window was applied to each frame

\footnotetext{
${ }^{1}$ The steps are: Expand the DTFT of the cross-correlation term, apply the noise properties to the expected values of the time domain samples, then express the result in terms of $\gamma_{x q}$ and $E\left[\left|Q_{w}[k]\right|^{2}\right]$.
}

and the result passed to a DFT. The Wiener gain was calculated and applied to each frequency sample. The result was inverse transformed, windowed again (same window) and applied to an overlap-add reconstruction. The system in the absence of gain modification is an identity system. The quantization noise energy was estimated from the step size of the quantizer. An oracle supplied the true signal magnitude to the calculation of the Wiener gain. The resulting SNR for the signal after postfiltering was calculated for a number of different signal levels and for a number of different values of $\gamma_{x q}$.

To the extent that the speech signal roughly follows a Laplace distribution, the correlation parameter should behave like the correlation parameter plotted in Fig. 2 as we scale the speech signal. That means that we would expect that for signal levels below that which maximizes the SNR, $\gamma_{x g}$ is positive, but less than one. We found that the best value of $\gamma_{x q}$ (a single value was used in the postfilter for the entire file) was well below unity, though it must be stated that the SNR value was only slightly increased as $\gamma_{x q}$ was changed from unity.

This experiment has its shortcomings. A better strategy would have been to choose a value of $\gamma_{x q}$ depending on the energy of the signal in each frame, rather than using a single value constant value. To do this properly will entail an extensive set of experiments to determine the functional relationship between the energy and $\gamma_{x q}$ (as in Fig. 2(b), but for speech signals).

\section{COMMENTS}

This paper has shown that quantizers that have pdf optimized output levels and/or have break points midway between output level will when the input signal level is matched to the design point of the quantizer, have a subtractive quantizer noise energy. For mismatched signals, the addition or subtraction of the quantization noise energy can be captured in a correlation parameter. This correlation parameter can vary widely, ranging from negative to positive values. This means that the quantization noise energy can be range from supersubtractive to super-additive. Indications are that postfiltering systems which try to mitigate the effect of the quantization noise should take this correlation into account. Further experimentation can determine algorithms which tune the Wiener gain, for instance, to the local correlation characteristics.

\section{REFERENCES}

[1] J. A. Bucklew and N. C. Gallagher, Jr., "A Note on Optimal Quantization", IEEE Trans. Inform. Theory, vol. 25, no. 3, pp. 365-366, May 1979.

[2] J. A. Bucklew and N. C. Gallagher, Jr., "Some Properties of Uniform Step Size Quantizers”, IEEE Trans. Inform. Theory, vol. 26, no. 5, pp. 610-613, Sept. 1980.

[3] N. S. Jayant and P. Noll, Digital Coding of Waveforms, Prentice-Hall, 1984 (ISBN: 978-0-13-211913-9). 